\title{
La subsunción indirecta del trabajo en el capital (su necesidad teórica)
}

\author{
Aquiles Montoya
}

En los paises del submundo capitalista la existencia de formas de producción no capitalista, no sólo los específica, sino que cualquier explicación de los mismos se vuelve insuliciente si no es capaz de dar respuesta al por qué de la persistencia de lales formas de producción no capitalista.

Nos parece que no es válido hacer a un lado el problema afirmando simplemente que estas formas de producción presentan una tendencia a la extinción, en lanto que la realidad socio-histórica nos está rechazado tal aseveración.

Tampoco nos resulta aceplable sostener que se trata de simples resabios de modos de producción previos, pero sin mayor importancia ya que vegetan como "los judios en los poros de la sociedad polaca". Lo cual, además de minimizar anlojadizamenle el problema, presupone la previa existencia de unos modos de producción que no pasan de ser meras suposiciones.

Igualmente nos resulta muy cuestionable el pretender explicar la existencia de formas de producción no capitalisla en tanlo que articulación o co-existencia de distinlos modos de producción, precisamente porque no se trata de distintos modos de producción sino tan sólo de disntinlas formas de producción.

También rechazamos las analogias de quienes pretenden explicar, por ejemplo, la producción campesina a partir de la subsunción formal del trabajo en el capital, en tanto no pasa de ser una simple analogía.

Mucho menos atinados nos parece las interpretaciones bipolares, tales como: la economía moderna y tradicional o la muy en boga: sector formal e informal; ya que no son capaces de llegar a la esencia de la realidad y se quedan en simples descripciones fenoménicas. Además de adolecer de una vaguedad tal, que pretendiendo abarcarlo lodo lerminan 
por no explicar nada. Nos parece que desde una perspectiva maxisla: un excelente principio epislemológico lo constituye el siguienle planteamienlo:

"En lodas las formas de sociedad existen una determinada producción que asigna a lodas las otras su correspondiente rango (e) influencia, y cuyas relaciones por lo tanto asignan a lodas las olras el rango y la influencia. Es una iluminación general en la que se bafian todos los colores y (que) modilica las particularidades de éstos. Es como un éter particular que delermina el peso especifico de lodas las formas de existencia que alli toman relieve". (1)

De la cita anterior, importa destacar en primer lugar, el reconocimiento explícito de la existencia de distintas formas de producción que efectúa Marx. En segundo lugar,..el carácter determinante que adquiere una. determinada Iorma de producción, al punto de asignar a las otras su correspondiente ranto e influencia. Tercero, de ello se deriva que la revelancia que puedan lener esas otras formas de producción estará subordinada a la producción que denominaremos principal. No se trata, pues, de producciones autónomas 0 independientes por muy articuladas que se quieran ver a la producción principal, sino de producclones subordinadas. Cuarto, serán precisamente las relaciones que se establecen al interior de esa producción principal las que aslgnarán a las olras su correspondiente rango e influencia. O sea que reconoce la existencia de otras relaciones de producción y de circulalación, pero que no son ajenas a la producción principal o subordinante, sino que por el contrario, su significación e importancia está determinanda por ella. En quinlo lugar, claramente habla Marx, no de eliminar las otras formas de producción y sus correspondientes relaciones, sino de modilicar su particularidad y serán modificadas, en mayor o menor grado, dependiendo del grado de desarrollo que alcance la producción subordinante y sus relaciones.

En los paises del submundo capitalista, -capilalista en tanto que la producción determinante es la producción capitalisla, y submundo en tanto que el rango y la influencia que poseen las formas de producción no capilalista es mayor que en los paises del mundo específicamente capitalista-, encontramos tres formas generales de producción: la producción capitalista, la producción mercanlil simple y la producción de valores de uso. Cada una de las cuales puede revestir formas particulares, no obslante, su comprensión resulla imposible si previamente no se logra explicar la relación existenle entre la producción capilalista y las formas de produción no capilalistas.

Somos de la opinión que ello es posible lograrlo a partir de la subsunción Indirecta del trabajo en el capital. Lo cual no dice nada, si 
previamente no recordamos los planteamientos que en torno a la subsunción formal y real efectúa Manx y si además, no esludiamos las formas de producción no capitalista haciendo abstracción de las formas particulares que estas revisten.

\section{A-Subsunclón (directa) del trabajo en el capital: formal y real.}

A nuestro juicio son tres los elementos que caracterizan la subsunción del trabajo en el capital. Tal como lo concibe Max; ya sea que se trate de la subsunción formal o real, a saber: a) inclusión, b) subor: dinación y c) relación directa entre el capilal y la fuerza de trabajo, la cual se compra y se vende en tanto que mercancia.

La inclusión hace referencia al hecho de que el proceso de trabajo se convièrte en el instrumento del proceso de valorización del capital. El proceso de trabajo se subsume en el capital, es un propio proceso. (2)

$x$ Respecto a la subordinación recordemos que el capilalista lo es entanto que personificación del capital, de allí que el trabajo está subordinado al capital en tanto que el capitalista se ubica en el proceso de trabajo como dirigente, conductor del mismo, ya sea velando porque " el Irabajo alcance el grado normal de calidad e intensidad" $\Varangle$ (3) ya sea con cambios reales en el proceso de trabajo, provocando una revolución lotal en los procesos de producción. La subordinación hace referencia también al carácter coercitivo de la relación entre el capitalista y el obrero, el cual es explotado por el primero debido a que no dispone de otro medio para vivir que no sea enlregando parte de su trabajo en forma gratuita al capitalista.

El proceso de trabajo incluido y subordinado, ésto es subsumido en el capital, "es al mismo tiempo de manera directa, un proceso de explotación de trabajo ajeno". (4) la cual se da mediante la relación salarial que se eslablece entre el capitalista y el obrero.

Sobre este lercer elemento Marx abunda en ilustraciones, asi plantea: "Cuando el campesino antafio independiente y que producia para su consumo se vuelve un jomalero.....; cuando (el) capitalista hace trabajar para si a los artesanos convertidos en asalariados; cuando el esclavista de otrora emplea como asalariados a sus ex-esclavos, etc., tenemos que procesos de producción determinados socialmenle de otro modo se han transformado en el proceso de producción del capilal" (5)

Además, de los tres elementos cualitativos previamente sefialados, Marx apunta un cuarto elemento de carácter cuanlitalivo; así plantea:

"Lo que distingue desde un principio al proceso de trabajo subsumido aunque solo sea tormalmente en el capital $-y$ por lo que va distinguiéndose cada vez más, incluso sobre la base de la vieja modalidad 
tradicional-, es la escala en que se efectúa; vale decir, por un lado la amplitud de los medios de producción adelantados, y por el otro la canlidad de los obreros dirigidos por el mismo palrón (employer)".(6)

O sea que no cualquier relación salarial conduce a una subsunción del trabajo en el capitat, aunque sea un presupuesto para la misma, es preciso que el empleador obtenga ingresos que posibiliten su consumo privado y constituir un fondo de acumulación, así como quedar libre del Irabajo directo, actuando tan solo como supervisor y director del proceso de producción, esto es, como capilalista, a tin de valorizar el valor primitivamente desembolsado.

Los anteriores elemenlos son los que a nuestro juicio caracterizan la subción directa del trabajo en el capital, ya sea que se trate de la subsunción formal o de la subsunción real. El elemento cuanlitalivo es clave a fin de distinguir cuándo estamos anle una producción capitalista o no, en tanto que aún en la producción mercantil simple puede enconlrarse trabajo asalariado.

Resulta necesario explicar por qué calificamos de directa a la subsunción del trabajo en el capital, cuando Marx no lo hace así.

Las razones que aducimos son las siguientes: em primer lugar, Marx sólo se ocupa dé relaciones de producción capitalista en donde el proceso de trabajo se ha convertido directamente en el intrumento del proceso de valóización del capital, donde la subordinación es directa y, obviamente la relación capital-Irabajo también es dlrecta, el obrero vende direclamente su fuerza de trabajo al capitalista. En tal sentido resultaba innecesario el calilicarla de directa. No obstante ello, en segundo lugar, hay suficienles elementos en los propios textos de Manx que nos evidencian el carácter directamente subsumido del trabajo en el capilal, asi en el primer párralo donde se ocupa de la subsunción formal dira:"... Para éste (el capilalisla) es al mismo tiempo, de manera directa, un proceso de explotación de trabajo ajeno". (7)

O bien, cuando señala: "El carácter distintivo de la subsunción formal del trabajo en el capital se destaca, con la mayor claridad, mediante el cotejo con siluaciones en las cuales el capital ya existe desempenando delerminadas funciones subordinadas, pero no aún en su función dominante, determinante de la lorma social general, en su condición de comprador dlrecto de trabajo y aproplador directo del proceso de producclón". (8)

Claramente sefiala que además de la compra direcla de fuerza de trabajo, hay una apropiación directa del proceso de producción. Trátase, pues, de una subsunción direcla del trabajo en el capital, la cual es estudiada bajo dor formas: la subsunción formal y la subsunción real. 
Cuando el capilal se apropia del proceso de trabajo, tal cual to encuentra, no tiene más salida que alargar la iornada laboral o bien, intenstficar el ritmo del trabajo a fin de incrementar la cusota de plusvalia por obrero, eslo es, acudir al mecanismo de la pulsvalía absoluta. En tal situación se dice que el trabajo está formalmente subsumıdo en el capital. En cambio cuando mediante un incremento en la capacidad productiva del trabajo se logra acortar la parte de la jornada laboral que corresponde al tiempo de trabajo necesario y como resullado de ello, se prolonga el tiempo durante el cual se crea la plusvalia, nos encontramos ante la subsunción real del trabajo en el capilal (9)

Tal incremento en la capacidad productiva, que implica un cambio real en el proceso mismo de trabajo, puede tener su origen en una variación de los métodos de trabajo, en los instrumentos de trabajo o en ambos a la vez.

Imporla también senalar cuándo es que Maxx considera que el trabajo no está siquiera formalmente subsumido en el capital. Presenta dos situaciones históricas, una referida al capital usurario que "adelanta en forma de dinero a los productores directos materias primas, instrumentos de trabajo o unos y otros, incluso. Los enormes intereses que obtienen; los intereses que, sea cual sea su monto, expolia al productor directo, no constituyen más que otro nombre de la plusvalía. Transforma su dinero en capital, de hecho, arrancándole al productor direclo trabajo impago, plustrabajo. Pero no se inmiscuye en el proceso mismo de la producción el cual hoy como ayer, se desenvuelve al margen de él a la manera tradicional.... Aquí aún no se ha realizado la subsunción formal del trabajo. en el capital. Otro ejemplo es el del capital comercial, por cuando hace pedidos a una serie de produclores direclos, reúne luego sus produclos y los vende; al actuar de esla suerte puede también adelantarles la materia prima, etc., e incluso dinero...... Tampoco en este caso estamos ante una subsunción formal del trabajo en el capital. El productor directo se manlienen siempre como vendedor de mercancias y a la vez como usuario de su propio trabajo". (10)

Parecen ser tres los elementos que Marx aduce para indicamos que en tales casos el trabajo no está subsumido formalmente en el capilal:

1. Que el capitalista no se inmiscuye en el proceso mismo de la producción.

2. Oue el productor directo se mantiene siempre como vendedor de mercancias, $y$

3. a la vez como usuario de su propio trabajo.

Consiguientememle, el capital no se ha apropiado directamente del proceso de producción, no es un comprador directo de fuerza de trabajo. 
Ahora bien, es importante percalarse que los ejemplos presentados por Marx corresponden a "situaciones en las cuales el capital ya existe desempenando determinadas funciones subordinadas, pero no aún en su función dominante de la forma social general......" (11)

No obstante, los elementos enumerados, nos parecen válidos para establecer cuando no existe siquiera subsunción formal del trabajo en el capital, pese a que ésle se haya constiluido ya en el delerminanle de la forma social general. Pero en esta nueva situación dirlamos que el trabajo no está subsumido directamente en el capital, aunque podria estarlo Indirectamente, en lanto que determinados procesos de trabajo esten subordinados indirectamente al capilal, se hayan convertido en inslrumentos de su valorización y no medien relaciones salariales. Tales serian las lormas de producción no capitalista, como la producción de valores uso para el autoconsumo y la producción simple de mercancias.

Estas lormas de producción no capitalista, bajo distintas modalidades particulares, se encuentran incluidas dentro de la lógica general del capital, esto es determin'das en su existencia y luncionamiento por las necesidades de valorización del capital. El capital mismo las ha convertido indirectamente en instrumentos de su propia valorización, al mismo tiempo que las subordina en tanto que exige posibilita su existencia, aunque lo haga directa o indirectamenle.

Las formas de producción no capitalista se encuentran subsumidas indirectamente en el capital a través de una relacion de expoliación, esio es, el mecanismo mediante el cual el capilal expropia-a los produclores no capitalistas de parte de su trabajo sin que exista entre ambos una relación directa en el proceso productivo.

Lo que diferenciarla a la subsunción directa_del trabajo en el capital de la indirecta es que en la primera la valorización se da mediante la compra de fuerza de trabajo, esto es, a partir de una relación salarial, en cambio en la indirecta el productor no produce bajo una relación salarial.

\section{B- La Producclón de valores de uso primer caso general de sub- sunción Indirecta.}

Cuando un proceso de trabajo culmina en un valor de uso y éste es consumido por sus productores, tal proceso de trabajo vislo desde su resultado será un proceso de producción de valores de uso y observado, desde su destino será una producción para el auto-consumo.

En tales condiciones el proceso de trabajo se convierte en un mecanismo de aulo-reproducción de la fuerza de trabajo. Ciertamente, la auto-reproducción que posibilita la producción de valores de uso no puede ser total, en tanlo que ninguna familia trabajadora está en condiciones 
de producir loda la gama de medios de vida que exige su reproducción.

Pero a su vez la auto-reproducción parcial de la fuerza de trabajo admite distintas gradaciones, dependiendo de muchísimos factores entre los cuales cabe mencionar: el monto de ingresos monetarios que perciba la familia, sea cual fuere el origen; la posibilidad real de producir valores de uso; el ámbito geográfico en el cual resida la familia; el grado de desarrollo de las relaciones de producción capitalista y el carácter particular que éstas revisten, etc.

Independientemente de la proporción en que se de la auto-reproducción de la fuerza de trabajo, lo imporlante es que nos encontramos con procesos de trabajo que se convierten en mecanismos de autoreproducción de la fuerza de Irabajo. Es la familia trabajadora, total o parcialmente, la que posibilita su auto-reproducción, valiéndose fundamentalmente de sus propios medios y, al hacerlo eslá produciendo una mercancia, la fuerza de trabajo que cuando se enfrenta al capital, que cuando se vende al capilal, no sólo genera un plusvalor fruto de la diferencia entre el valor nuevo producido y su propio valor, sino que el salario que percibe es inferior a su valor, con lo cual posibilita al capilal una valorización extraordinaria.

Comprender este punto que pareciera de diticil demostración, resulta realmente sencillo, basta con reparar en que si la luerza de trabajo se pagase por su valor, con lodo lo que ello implica, la farnilia trabajadora no necesitaría recurrir a la producción para el auto-consumo.

De alli que una prueba que se puede aducir para demostrar que la fuerza de trabajo no se paga por su valor es la existencia, no casual, ni fortuita sino sistemática y permanente, de procesos de producción para el autoconsumo.

Estos procesos de trabajo que se convierten en mecanismos de autoreproducción de la fuerza de trabajo pudiera creerse que son independientes de la producción capilalisla, que no tienen nada que ver con la lógica del capital. $O$ en lodo caso que es una suerte para el capital, o los capitalistas, el encontrarse con una fuerza de trabajo parcialmente autoreproducida y, en esa medida, sin que les signitique pagar todo su costo.

Ciertamente desde la perspectiva que venimos analizando esta relación entre el trabajo y el capital, lo que cuesta la producción y reproducción de la fuerza de trabajo y lo que le cuesta al capital, se nos revelan como dos magnitudes diterentes, la razón de ello la encontramos en el hecho de la auto-reproducción de la fuerza de trabajo, la cual declamos dá lugar a una valorización extraordinaria del capital.

Esla valorización extraordinaria del capital es de suma imporlancia para comprender que no se trata de procesos de trabajo ajenos a la 
Kogica del capital, sino que por el contrario es el capital, el que exige y posibilita por distintos medios la auto-reproducción de la fuerza de trabajo.Para cierla fracción de la clase trabajadora la producción para el auto consumo se convierte en una exigencia, ya sea debido al carácter estacional de su ocupación, al bajo nivel de sus ingresos salariales, al mínimo nivel de ocupación que posee, al elevado grado de sustitutibilidad, etc., factores todos ellos que, directa o indirectamente, son determinados por el capital y a su vez es el capital, el que directa o indirectamente hace posible la producción para el auto-consumo; por todo ello es posible sostener que estos procesos de trabajo que se convierten en mecanismos de auto-reproducción de la fuerza de trabajo, lejos de ser ajenos a la lógica del capital se encuenlran subordinados a la producción capitalista.

En cuanto a lo que es el proceso de trabajo como producción para el auto-consumo, el capital o su personilicación: el capitalista, no establece ninguna relación directa con el trabajo, como trabajo asalariado. son los produclores solos o auxiliados por instituciones no capitalista las que proyectan, ejecutan y controlan el proceso de trabajo. En cuanto a los medios de producción pueden ser o no de su propiedad, no osbstante los productos oblenidos si lo son, aunque tengan que compartirlos, en el caso de que los medios de producción no les pertenezcan.

No obstante el último elemento sefialado mediamle el cual si podría establecerse una relación directa con el capital, es pertinente sefialar que no se trala de una relación directa entre el trabajo y el capital, el productor no se vincula con el capital como Irabajo asalariado, no le vende su fuerza de trabajo, sino que le compra o alquila medios de producción; o bien, recibe dinero en préstamo del capital o del Estado.

De to anleriormente expuesto resulta posible eslablecer algunas conclusiones de suyo muy importante para comprender y explicar la presencia y permanencia de procesos de producción de valores de uso o procesos de producción para el auto-consumo.

a) Observámos que tales procesos de Irabajo en la medida que se convertian en mecanismos para la auto-reproducción de la luerza de trabajo, en la misma medida se convertian en instrumento de valorización extraordinaria del capital.

b) También estableciamos que los mismos procesos de trabajo se encuentran subordinados al capital en tanlo es éste el que exige y posibilita su realización.

c) Además senalábamos que no media una relación directa entre el trabajo y el capital en el proceso de producción de valores de uso. 
d) Se desprende también de lo expuesto, aunque no fue explicitado, que la relación a que dan lugar eslos procesos de trabajo, cuando la fuerza de trabajo entra en contacto con el capital, es una relación doble, por un lado es una relación de explotación y por otro, una relación de expoliación, la primera da lugar a la apropiación de plusvalia y la segunda, a una plusvalía extraordinaria.

Este conjunto de elementos encontrados en el proceso de producción de valores de uso en la producción para el auto-consumo nos llevan a sostener que en tales casos lo que se da es una subsunción indirecta del trabajo en el capital.

O dicho en términos más generales, cuando el proceso de trabajo derive en producción para el aulo-consumo, en tanto es un mecanismo de auto-reproducción de la fuerza de trabajo se encuentra subsumido indirectamente en el capital. Siendo la expoliación o el intercambio entre no equivalenles, la relación que caracteriza a los procesos de trabajo subsumidos indirectamente en el capital.

La subsunción indirecta del trabajo en el capital tal como ha sido expuesta incluye, pero sin reducirse a ella, la realidad contenida bajo el tílulo de "subsunción marginal" de Veronika Bennholdt Thomsen, quien planlea: "Marginalmente subsumidos están todos aquellos productores agrícolas que se emplean en cualquier actividad, sin tener un trabajo especilico con el que podrian obtener los medios de vida para su reproducción". (12) Más adelante agrega: "Si encuentran un trabajo asalariado, el pago es extremadamente bajo.....; por olro lado esta forma extrema de explolación de trabajo asalariado sólo es posible porque obtienen sus medios de vida de otro proceso de trabajo, es decir, en su propia producción, producción minifundista".(13)

Aunque es importante sefalar que la autora antes cilada ve subsunción lan solo en cuanto estos productores se convierten en Irabajadores asalariados, más no en el "olro proceso de trabajo", aquel de "su propia producción"; nosotros en cambio observamos la subsunción indirecta del Irabajo en el capital precisamente en ese proceso de producción; es ese trabajo productor de valores de uso el que está subsumido indirectamente en el capital. (*)

Roberlo Rubio, quien dedica un aprtado especial a la subsunción indirecta del trabajo en el capital (14) no tiene ninguna referencia a la producción de valores de uso; tampoco nos ha aparecido en los pocos autores que consideran la subsunción indirecla, tales como: Win Diercksens, Erneslo Richler, Michel Beaud, Bertrand Bellon y Patrick Francois. (*)

\footnotetext{
- Veáse su trabajo citado en la nota anterior.
} 
La explicación posible para ello es que, en general, parten de las modalidades particulares que revisten las formas de producción no capitalistas, eslo es, pasan a ocuparse directamente de la producción campesina, de la producción artesanal, elc.

A nosotros en cambio, como ya lo senalábamos, nos parece correcto comenzar estudiando las lormas básicas de producción no capitalista. Ciertamente, la producción de valores de uso para el aulo-consumo no se presenla en forma pura, puede efectuarla el productor agricola, como puede efectuarla el atesano, o el trabajador domiciliario urbano o rural, como también puede hacerla un asalariado permanente o estacional , etc. Puede ser más o menos significativa para la reproducción de la fuerza de trabajo. Asl como ocasional o permanente. De alli que iniciar su estudio a partir de las particularidades, nos parece un camino no debido, ya que lo más probable es que ó nos hubiéramos perdido con tal cúmulo de siluaciones particulares o hubiéramos terminado por hacer abstracción de las mismas, pero sin lograr aproximarnos a lo concrelo, como unidad de lo diverso.

\section{C- La producción simple de mercanclas segundo caso general de subsunclón Indirecta del trabajo en el capltal.}

Cuando el proceso de trabajo culmina en un valor de uso cuyo destino es el intercambio, ese producto del trabajo revisle la lorma mercanla. "El produclo del Irabajo es objelo de uso en lodos los tipos de sociedad; sólo en una época históricamente dada de progreso, aquella que ve en el trabajo invertido para producir un objeto de uso una propiedad materializada de esle objeto, o sea su valor, se convierte el produclo del trabajo en mercancía". (15)

La mercancia se nos presenta como la unidad contradictoria de valor de uso y de valor, fruto de un mismo proceso de trabajo, que se desdobla en trabajo concreto, generador de valor de uso, al considerarlo cualitativamente $y$ en trabajo abstracto, generador de valor, al considerarlo cuantitativamente.

La diferencia entre la producción simple de mercancias y la producción capilalista de mercancías radica en que, "Como unidad de proceso de trabajo y proceso de creación de valor, el proceso de producción es un proceso de producción de mercancías; $(y)$ como unidad de proceso de trabajo y de proceso de valorización, el proceso de producción es un proceso de producción capitalista, la forma capitalisla de producir mercancias". (16).

Veáse : "Polltica y población" de Diercksens; "Proceso de acumulación y dominación en la lormación socio-polltica salvadoreña" de Pichter y "Para leer el capitalismo" de Beaud. Bollon y Francois. 
El por qué un proceso de producción encierra un proceso de valorización y el otro no, reduciéndose a una simple creación de valor, deriva de las relaciones de producción. En la producción capitalista, el produclor trabaja para olro, en la producción mercantil Irabaja para sí. El produclor direclo en el régimen capilalisla no es propietario de los medios de producción, ni de los trulos de su trabajo, ni controla el proceso de trabajo; al vender su fuerza de trabajo para que la use otro con el fin de valorizar el valor primitivamente desembolsado, su trabajo eslá directamente subsumido en el capilal.

Lo propio de la producción mercantil seria que el productor directo conserve la propiedad, o bien, la simple tenencia de las condiciones de producción y de los medios de subsistencia; que controle directamente el proceso mismo de producción y que siendo usuario de su propio trabajo, venda los productos del mismo y no su fuerza de trabajo.

En la producción mecantil es común observar participando a la par del productor principal a su familia, a aprendices, e incluso jornaleros o asalariados, pero en una cuantía tal que no transforma la naturaleza de la producción. Recordar el elemento cuantitativo sefialado a propósito de la subsunción directa del trabajo en el capital.

Si bien el destino de la producción es el intercambio, su finalidad es la auto-reproducción como unidad productora y de esta manera garantizar la existencia y reprodución de la familia, base de la producción mercantil.

Los productos obtenidos, por su naturaleza, pueden ser empleados como medios de producción y como medios de vida, de alli que una fracción de los mismos pueden reintegrarse en el siguienle proceso de producción, o bien emplearse como medios de vida, posibilitando así la auto-reproducción de la fuerza de trabajo, con lodas las implicačiones sefialades en el apartado anterior, si es el caso que esta fuerza de trabajo auto-reproducida se venda al capilal. Esto sería en tanto que la producción mercantil incluye en si una simple producción de valores de uso y en esa medida es asimilada al primer caso general de subsunción indirecla, pero lambién en lanto productora y realizadora de mercancias posibilita la auto-reproducción de fuerza de trabajo sin costo alguno para el capital, con lo cual se convierle en un instrumento de su valorización, lo cual seria suficiente para que el capital posibilitara y/o exigiese la existencia de esla forma de producción no capitalista. Pero no se reduce a ello la función que cumple la producción mercantil, tambien constiluye un relugio temporal o permanente para la fuerza de Irabajo que no requiere el capilal, sin cargar los coslos que ello implicaria de no existir tal lorma de producción no capitalista.

Esto por lo que se refiere a la producción mercantil en tanlo que uni- 
dad productora y reproductora de fuerza de trabajo "barata" para el capital.

Detengámonos ahora en los productos en tanto que mercancias y en las relaciones que contraen los productores mercantiles, previas al proceso de producción como al linal del mismo. Las mercancías por su naluraleza y en tanto productos, separables de sus productores, ésto es con existencia corpórea, pueden ser medios de vida o medios de producción. Sus destinatarios finales serian: otros productores simples de mercancias, los capitalislas y los asalariados. Entre los productores y los consumidores finales pueden presentarse intermediarios, tales como: capitalistas, simples comerciantes y el Estado. A fin de hacer posible el proceso de producción, los produclores tienen que entrar en relación con otros productores mercantiles, con capilalistas, con el Estado, con Comerciantes y con otras instituciones. Es de suma importancia el develar estas relaciones en tanto que son ellas las que posibilitan comprender la exislencia y desaparición de la producción mercantil simple.

Antes de proceder al estudio de las principales relaciones de los productores mercantiles es preciso percatarnos que ellas se dan en un mercado dominado por la lógica del capital, no obstante, los productores mercantiles no operan con esa lógica, ni al producir, ni al realizar sus mercancias; influídos por la lógica capitalista podrian pretender actuar conlorme a ella, pero les resullaria imposible porque entonces tendrían que actuar como capitalistas y no cuentan con los recursos ni los medios para hacerlo, de allí que terminarian por ser destruidos. El produc-tor mercantil sólo puede subsilir en tanto que actúe como tal, llenando vacios dejados por el capital, complementando la producción capitalisla y en tanto sea tolerado por el capital; en general, en la medida que su exislencia resulte beneficiosa para el capital.

El actuar como tal productor mercantil implica que al realizar sus mercancias puede aceptar, y de hecho acepla, un precio que es considerablemente interior al valor de su producto, o inferior al costo de producción. Surge la interrogante, ¿Por qué el productor mercanlil cantinúa produciendo en tales condiciones? Responder que ello es debido a que no opera con la lógica del capilal, no dice nada nuevo, ello ya lo sabemos.

La respuesta radica en que el productor mercantil no posee otra alternativa; o realiza su producción en esas condiciones o sencillamente no produce, con to cual pone en peligro su sobrevivencia. Es la necesidad de sobrevivir, entonces lo que le obliga a aceptar unos precios interiores al valor de su producto, o inferiores al costo de producción. Si obtiene precios iguales al costo de producción, habrá oblenido un equivalente al valor de su fuerza de trabajo, con lo cual se estaria 
asegurando su reproducción y la de su familia. Si fuese el caso de que sacrifica parte de sus coslos de producción, siempre puede seguir produciendo, ya que le resultaria peor no obtener ningún ingreso, aunque ésto sólo fuese una tracción de lo que obtendria de vender su fuerza de tra-bajo. Pero sucede que es precisamente la imposibilidad de vender su fuerza de trabjo en forma permanente lo que le obliga a ser un productor mercantil. Lo anterior nos brinda los elementos fundamentales para comprender la existencia y desaparición de la producción mercantil, por un lado está la conveniencia para el capital y el Estado de su existencia en tanto provee medios de producción y de vida a precios inferiores a los que exigirian los produclores capitalistas. Pero a su vez está la conveniencia para el productor mercantil de asalariarse y en tanto pueda hacerlo, lo hace.

Pasemos ahora a ocuparnos de las principales relaciones de los productores mercantiles:

a) La producción mercanlil y el Estado

En primer lugar es preciso dejar establecido que el estado en una economia capilalista responde a la lógica del capital y en esa medida su principal función es asegurar la reproducción del capital. Ciertamente sus personificaciones, los capitalislas, no son una unidad homogénea, lo cual puede conducir a engaño en lanto que pueden presentarse conIradicciones entre el Estado y ciertas fracciones de la clase capilalista, pero bien mirada la situación particular, se descubrirá siempre que el lin mediato está acorde con los intereses del capital.

El Estado provee a los productores mercantiles básicamente de dinero, insumos, asislencia técnica (gratuita) y medios de vida a precios inferiores de los que pagarían los productores mercantiles de obtenerlos de otras fuentes. Lo anterior no quiere decir que todos los productores mercantiles reciban del Estado, los cuatro elementos senalados, mucho depende de la naturaleza de la producción y del produclor mismo. Importa si retener que hay un interés de parte del Estado por contribuir a la permanencia de la producción mercantil.

Por otra parte tenemos que el Estado demanda parte de la producción mercantil de origen agrícola y al hacerlo es éste quien fija el precio de compra.

Cuando se trala de bienes alimenticios, el interés primordial del Estado es lograr mantener bajos sus precios, a fin de contribluir en forma significativa a mantener bajo el coslo de reproducción de la fuerza detrabajo y en esa medida el capilal puede manlener bajos sus costos via salarios, con lo cual se le posibilita una mayor valorización, en lanto se incrementa la cuota de explolación por trabajador asalariado. 
También puede resultar beneficiado el capital cuando participa en la producción de la misma mercancia cuyo precio tija el Esatado, si esle precio cubre el coslo de producción mercanlil y la produclividad de la empresa capitalista es considerablemenle superior, enlonces puede apropiarse de una plusvalia ex/raordinaria.

En cuanto a la producción mercantil no agricola la participación del Estado eslá más claramenle orienlada a posiblitar su surgimiento y preservación, a través de proveer dinero en préslamo a eslos productores, asi como algún tipo de asesoria; la razón particular cuando se trala de medios de vida sería abaratar la fuerza de trabajo, dado que como estableciamos, la produción mercantil en general realiza sus mercancias a precios interiores a su valor y tratándose de mercancias que son a su vez producidas por el capital no tienen más allernativa que vender a precios inferiores que aquellos de los labricantes capilalislas.

Además de los elementos sefialados, el Estado tiene un interés de carácler más general en cuanto a la existencia de la producción mercantil, debido a las olras funciones que cumple esta producción, sefialadas anleriormente, cuales son: posibililar la auto-reproducción de la fuerza de trabajo, asi como su auto-conservación para cuando el capital la necesite y un refugio para la misma cuando el capital ya no la requiere. Al acluar de esta manera el Estado, claramenle está respondiendo a la lógica del capital: posibilitándole una mayor valorización y a su vez asegurándole la existencia de una fuerza de Irabajo apta para ser usada cuando la requiera, así como descargándose y en esa medida descargando al capital, de los coslos de manlenimienlo de una fuerza de trabajo ya inúlil para el capital. Inútil para el capilal, pero no inútil en sí misma, lo que sucede es que el capital prefiere usar la fuerza de trabajo en su edad mucho más productiva, de alli el alto grado de sustilutibilidad que sutre la misma, cuando se dan las condiciones antes sefialadas.

b) La producción mercantil y los asalariados, otros productores mercantiles y los comerciantes.

Las relaciones que establecen los productores mercantiles con los asalariados, con otros produclores mercantiles y con los comerciantes se dan a nivel de la circulación y poco o nada inciden en el proceso de producción mismo. En general son relaciones de compra-venla en donde el dinero opera como simple medio de cambio.

Los asalariados obtienen de los productores mercantiles medios de vida y muy ocasionalmente, algún medio de producción a lin de electuar ciertas actividades produclivas para el auto-consumo. Las implicaciones de esta relación ya han sido develadas con anterioridad, acá, tan solo interesa destacar que existe también una relación directa entre el productor mercantil y el asalariado. 
En cuanto a la relación que se da entre productores mercantiles, importaría percatarse que al proveerse entre sl de medios de producción o de vida, se lacilitan mutuamente su reproducción como lales, con todas las implicaciones ya consideradas.

Por b que hace a las relaciones que contraen con los comerciantes importa destacar que la función de éstos es la de servir de intermediarios entre los productores mercantiles y los capilalislas y/o los asalariados. Aunque ciertamente el comerciante compra para vender más caro, el diferencial que obtiene más que constituir un beneficio, es un ingreso que bien equivale a lo que oblendria de vender su fuerza de trabajo. Nos parece que el comerciante, al igual que el productor mercantil no opera con la lógica del capital, en muchos casos ni siquiera son propietarios del patrimonio dinero con el que compra las mercancias. Pensamos que parcialmente su existencia misma responde a la existencia de la producción mercantil.

Si bien el comercianle se queda con parte del valor producido por el productor mercantil, al no exigir para sí una ganancia media y poseer costos menores que el capital comercial, puede realizar las mercancias producidas por el productor mercantil a precios menores que el capital comercial, con to cual estarla contribuyendo al efecto ya senalado de "abaratar" la fuerza de trabajo.

Pero el comerciante también puede ser el medio a través del cual el capital obtienen materias primas de los productores mercantiles ¿Beneticiará tal intermediación a los capilalislas? Nos parece que si, en tanto que el capitalista en particular se ahorra tiempo y recursos al tratar directamente con unos pocos vendedores, sin tener que pagar más de lo que pagarla a los productores mercantiles de tratar directamente con ellos. Además, lal intermediación del comerciante le hace parecer como que es él quien expolia a los productores mercantiles, cuando tan solo es un instrumento al servicio del capital, un medio posibilitante de una mayor valorización del capital en cualquiera de los dos casos sefialados.

c) La producción mercantil y el capital.

En los anteriores apartados aunque no se ha tratado de la relaciones entre la producción mercantil y el capital, éste ha eslado siempre presente y baslaría con lo anterior para aceptar que la producción mercantil, responde a la lógica del capital, que es éste quien determina su existencia, a la vez que la condiciona. Que la producción mercantil es un instrumento de valorización del capilal y que en esa medida éste posibilita y exige su existencia.

Pero importa aún esclarecer algunas relaciones de suyo muy importantes que pueden incluso afectar a la naturaleza de la producción mercantil misma. 
c.1 Veamos primero las relaciones que establecen los produclores mercantiles, en tanto demandantes, con el capital.Ciertamente habrá dos vinculaciones a nivel de la circulación: con el capital comercial y con el capital bancario. El capilal comercial suministra a los productores mercantiles aquellos medios de vida y de producción que éstos no pueden adquirir de otras luenles, tal relación es una relación de compra-venta, en donde ambos, se enfrentan como sujelos independientes. No existe ningura relación especial entre el capitalista comercial y el productor mercantil, uno vende y el otro compra. Para el capilalista comercial en particular lo mismo le da venderle al productor mercantil, que a un asalariado o a otro capilalista. Sin embargo, los capilales comerciales exigen para si una delerminada lasa de ganancia, asl venden sus mercanclas a precios que oscilan en tomo al precio de producción, en tanto que capilalistas aclúan conlorme a la lógica del capilal. Los productores mercantiles por su parte, no pueden actuar con tal lógica, pero en lanto se ven obligados a comprarle a los capitalistas comerciales, no tienen más alternativa que someterse a la lógica del capital. Lo anterior lleva a sostener que el capital comercial expolia a los productores mercantiles, via un intercambio desigual, ya velamos que las mercancias son adquiridas por los productores mercantiles a precios que corresponden aproximadamenle a sus precios de producción, en cambio cuando vende los propios lo hacen a precios inferiores a su valor. No obstamte, el problema que deseamos senalar es más radical y deriva del hecho de que los productores mercantiles se ven cada vez más constrenidos a demandar mercanclas producidas en forma capitalista, se ven inmersos en un mundo dominado por la logica del capital, sin poder actuar ellos mismos con esa lógica, lo cual tiene algunas implicaciones: primero, sus patrones de vida y consumo, así como sus procesos mismos de producción se ven afectados por la producción capitalista. Algunos ejemplos: la necesidad del radio, el uso de artículos de plástico, empleo de fertilizanles y herbicidas, productos sintéticos, elc.; segundo, al no poder realizar sus mercanclas por su valor se ven obligados a sacrificar cada vez más la parte que les corresponderla como remuneración a su trabajo o bien a trabajar más tiempo y/o más intensamente y al hacerlo, arrastran consigo a quienes les auxilian en el proceso de producción; tercero, el resultado de todo ello redunda en un mayor beneficio para el capital como un todo, en tanto no sólo se amplla el ámbito de realización de sus mercanclas, sino que a su vez se da una mayor expoliación del productor mercantil. 
Si se dejara a los fenómenos anteriormenle descritos desarrollarse expontáneamenle la resultante no podria ser otra que la desaparición del productor mercantil, a lin de contrarrestar tal tendencia se hace presente el estado con sus politicas de apoyo y lomento a la denominada "microempresa", al igual que otras insfituciones privadas financiadas generalmenle con fondos externos.

Respecto al capital bancario su relación con la producción mercantil no es significativa ya que la generalidad de los pro-ductores mercantiles no son "sujelos de crédito", cuando reciben algún financiamiento es de parte de instiluciones eslatales, las cuales ya fueron estudiadas en el aparlado correspondienle.

c.2 Nos toca ahora ocuparnos de las relaciones entre la producción mercantil y el capital, en tanto que demandante de las mercaderias producidas por aquella. El capilal se nos presenta bajo dos modalidades, la del capilal industrial que demanda medios de producción y el capital comercial que demanda, principalmente. bienes de consumo. Tanto el uno como el otro se caracterizan por poseer una mayor capacidad de negociación frente a los productores mercantiles, por ello lerminan siendo ellos quienes rijan el precio de compra de las mercancias; precios que como ya veiamos y por las razones tambien sefaladas, son inferiores al valor de las mercancias, 0 al costo mismo de producción. En tanto que los produclores mercantiles se relacionen con distintos capitalistas, sean eslos industriales o comerciales, buscando obtener el mejor precio posible para sus mercancias, no se establece entre ambos más que simples relaciones de compra-venla de los productos de su trabajo. Cierlamenle hay una expoliación del productor mercantil por parte del capitalista particular con el cual se relaciona. Expoliación que posibilita al capitalisla una mayor valorización de su capital, en tanto que él si exige para si, al menos la tasa media de ganancia. Pero además, en la medida que los capitalistas se proveen en magnitudes considerables a través de productores mercantiles, en lanto éstos son expoliados, el valor del cual expropian a los productores mercantiles y que en sus manos se convierten en plusvalla, en esa media se les posibilita contrarrestar la caída de la tasa de ganancia.

Ocasionalmente, el capital industrial o el comercial pueden adelantar dinero a los productores mercantiles, lo cual se convierte en una fuente adicional de expoliación a través del interés que exigen por el dienero adelantado. Interés que ciertamente será superior, o al menos igual, a la tasa comercial. Pero aquí eslos capitales están actuando más como capital bancario o financiero, 
que como capital comercial o industrial y si el produclor mercantil acude a ellos es precisamente porque no ha lenido acceso a olra luente de linanciamiento.

También en forma ocasional pueden adelantarle al productor mercantil medios de producción, ya sea insırumentos de Irabajo y materias primas 0 insumos agricolas. También aqui hay lugar para una fuente adicional de expoliación, en tanto la venta al crédito posibilita establecer un precio superior al ordinario; si el productor mercantil acepta tal situación es porque no posee otra alternaliva menos onerosa.

En las dos últimas siluaciones planleadas queremos destacar que se Irala de relaciones ocasionales, fortuitas, aunque ciertamente derivan de la relación de subordinación en que se encuentra la producción mercantil Irente al capital, más no frenle a ciertos capitalistas en particular, cuales serian los sefialados previamente. Es el impaclo que la producción capitalista ha ejercido sobre ellos los que les lleva a suslituir el antiguo instrumento de trabajo, sin tener la capacidad, pero si las necesidades de hacerlo a fin de incrementar la productividad de su trabajo; o bien, al uso de semillas mejoradas, de lertilizanles y de herbicidas que aquellos exigen, elc.

O bien al uso de nuevos tipos de materias primas a fin de poder realizar sus mercancias, etc.

Con todo, conservan su calidad de productores mercantiles en tanto los capitalistas particulares con los cuales se relacionan a través de la venta de sus mercancias, no se inmiscuyen en forma alguna en el proceso de producción mismo. No se trata de una producción por encargo y en esa medida no hay una especilicación de parte del capilalista en particular respecto al producto.

Cierlamente, en la relación directa de los productores mercantiles con el capital, se observa en forma más clara cómo sus procesos de trabajo se convierten en instrumentos del proceso de valorización del capital, auque lal fenómeno ha sido constante, sin que el productor mercantil se relaciones directamente con el capital, como hemos intentado mostrarlo a lo largo del estudio de la producción mecantil. Por consiguiente, loda producción mercantil eslá incluída aunque de manera indirecla en la reproducción del capital; en lanto posibilita una mayor valorización del mismo. Pero a su vez, aunque no se de una subordinación directa a determinados capitalistas particulares, toda la producción mercantil está subordinada indirectamente al capital como un todo, no solo en tanto posibilita y exige su permanencia - aunque to haga indirectamente a través del Eslado u otras instiluciones-, sino también en cuanto modifica los patrones de producción y de vida de los productores mercantiles, en 
esta forma está determinando su manera de existencia particular, de allí que las formas especlficas en que se presenta la producción mercantil como podrlan ser los campesinos, los artesanos, los Irabajadores domicjliarios, etc., están radicalmente modificadas; la subordinación también se nos revela cuando reparamos en qué producción mercantil se tolera o fomenta; y todavia más, su carácter subordinado es evidente al no poseer autonomia en cuanto al precio de realización de sus productos, ya sea porque el Estado interviene fijando precios o porque la producción capilalista no les deja otra alternativa de sobrevivencia que vender a precios inferiores al costo de producción. Igualmente se nos revela su carácter subordinado atendiendo a la función que cumplen respecto a la producción, conservación y manlenimiento de la fuerza de trabajo, antes, durante y después de que la requiera el capital. Por tanto, la producción mercantil simple está subsumida indirectamenle en el capital en lanlo es un instrumento de valorización del capilal y en tanlo está subordinada al mismo.

En sintesis los elementos que nos senalan el carácter indirectamente subsumido de la producción mercantil al capital son las siguienles:

a) En tanto que la producción mercantil incluye a la producción de valores de uso, pero sin reducirse a ella, en lodo lo que implica producción para el auto-consumo es aplicable to establecido respecto a la subsunción indirecla de la producción de valores de uso al capital.

b) La producciónn mercantil al asegurarse ciertos ingresos resultantes de la realización de sus mercancias posibilita la auto-reproducción de la luerza de trabajo sin costo alguno para el capital, con lo cual se convierte en un instrumento mediato de valorización del capital.

c) La producción mercantil al realizar sus mercancias a precios inleriores a su valor, o inferiores a su costo de producción, se convierte en un instrumento de valorización extraordinaria del capital. Ya sea cuando produce medios de vida para los asalariados o cuando produce medios de producción para los capitalistas, en ambos casos contribuye a disminuir los costos del capital y consecuenlemente, incrementar sus beneficios. Además de otras situaciones de carácter menos general.

d Además de los elementos anteriores en los cuales se sefiala que la producción mercantil se convierte en un instrumento del proceso de valorización del capilal y, por lanto, está incluída dentro del proceso global de auto-valorización del capital, sucede que la producción mercantil sólo puede subsistir en tanto actúe como tal, siendo determinada en su existencia o desaparición por las 
necesidades del capital -aunque para posibilitar su existencia o surgimiento se valga de la particpación del Estado-, en tal sentido la producción mercantil está subordinada al capilal.

e) Pero la subordinación de la producción mercantil al capital no se revela solamente en su existencia, sino en la forma misma de su existencia, la producción mercanlil va siendo modificada en su como producli por la producción capitalisla la cual le abastece en mayor o menor medida de medios de producción. Igualmente, le moditica el tiempo y la intensidad de su trabajo. E incluso llega a modificarle sus necesidades y los satislactores de las mismas.

Por tanto, si la producción mercantil es un instrumenlo del proceso de valorización del capilal, a través de las distintas vias sefaladas, y consiguientemente incluida denlro del proceso de valorización del capital como un todo, tenemos un primer elemento para sostener la subsunción de tales procesos de trabajo en el capital. Pero si además de ello la producción mercantil, sin dejar de serlo, está delerminada en su existencia y en su forma misma de existencia por la lógica del capital, consecuentemente está subordinada al capital y por tanto lenemos un segundo elemento para soslener que lales procesos de Irabajo se hayan subsumidos en el capital.

Existiendo inclusión y subordinación pero no una relación salarial enre estos procesos de trabajo y el capital, lo que tenemos consiguientemente es una subsunción Indirecta, medlata de la producclón mercantll en el capltal. La cual se caracteriza por la expoliación de los trabajadores en tanto auto-reproductores de su luerza de trabajo y vendedores de mercancias. Esta expoliación de valor de que es objeto la producción mercantil, se convierte en plusvalia en manos del capital. Pero a su vez la luerza de trabajo generada en el seno de la producción mercantil, al entrar en contacto con el capital, da lugar a una doble relación: una relación de expoliación al comprar una fuerza de trabajo sin pagar sus costos de producción y una relación de expoliación en tanto generante de plusvalia durante el tiempo que es utilizada.

Por tanto, cualquier torma particular en que se encuentre la producción de valores de uso o la simple producción mercanlil, ya sea en forma pura o combinada, se haya subsumida indirectamente en el capital, que lenga de producción de valores de uso o de producción mercantil. Es pues la subsunción indirecla del trabajo en el capital la que explica la existencia de las formas no capilalistas de producción, cualquiera que sean las modalidades particulares que revistan, como son: producción doméstica, producción campesina, producción artesanal, producción domiciliaria o bien formas híbridas, tales: producción doméstica y trabajo asalariado, producción campesina y trabajo asalariado, elc. 
Aunque es claro que en las formas híbridas habria subsunción directa $e$ indirecta, las cuales pueden darse en forma alterna o simultánea, dependiendo del caso particular.

\section{NOTAS}

1. Marx, Kart. Elementos Fundamentales para la Critica de la Ecoriomia Pollica. (borrador) 1857-1859. Vol. I

Editorial Siglo XXI. Máxico 1971. Pag. 28.

2. Marx, Kart. El capital, Capitulo sexto (Inédito)

Editorial Siglo XXI. Máxico, 1979. Pag. 54

3. Ibid. Pag. 55

4. Ibid. Pag. 54

5. Ibid. Pag. 54

6. Ibid. Pag. 57

7. Ibid. Pag. 54 (Subrayado nuestro)

8. Ibid. Pag. 58 (Subrayado nuestro)

9. Ibid. Pag. 59

10. Ibid. Pag. 58

11. Ibid. Pag. 58

12. Bennholdt Thomsen, Verónica "Problemas en el Análisis de Clases en el Sector Agrario en palses dependientes:

Cuadernos Agrarios, Año 2, No. 5. México 1977, Pag. 32

13. Ibid. Pags. $32-33$

14. Rubio, Robert. Las formas generales de dominlo del capltal sobre ef trabajo dentro de la agheultura ealvadorefie. Mimeografiado. Tésis de Licenciatura en Economla. UCA. EI Salvador, 1979.

15. Marx, Kari. El Capital, Tomo I.

Editorial FCE, México, 1979. Pag. 28

16. Ibid. Pag. 147 